\title{
Properties of GaN Nanocolumns Grown by Plasma-Assisted MBE on Si (111) Substrates
}

\author{
Z.R. Zythiewicz ${ }^{a}$, P. Dluzewski ${ }^{a}$, J. Borysiuk ${ }^{a}$, M. Sobanska ${ }^{a}$, K. Klosek $^{a}$, \\ B.S. Witkowski ${ }^{a}$, M. Setkiewicz ${ }^{b}$ And T. Pustelny ${ }^{b}$ \\ ${ }^{a}$ Institute of Physics, Polish Academy of Sciences, al. Lotników 32/46, 02-668 Warsaw, Poland \\ ${ }^{b}$ Silesian University of Technology, Akademicka 2, 44-100 Gliwice, Poland
}

\begin{abstract}
We report on growth of GaN nanocolumns by plasma assisted MBE on (111) silicon substrates and on their characterization. The nanocolumns nucleate on the substrate spontaneously without use of any catalyst, probably by the Volmer-Weber mechanism. Transmission electron microscopy analysis shows high crystalline quality of GaN nanocolumns and their good alignment with the $c$-axis being perpendicular to the substrate. Preliminary results on use of GaN nanocolumns in gas sensor devices are presented.
\end{abstract}

PACS: 81.15.Hi, 81.05.Dz, 61.46.Km

\section{Introduction}

Due to their unique electronic properties and easy relaxation of lattice strain leading to high crystallographic perfection even when grown on lattice-mismatched substrates, semiconductor nanocolumns (NCs) are ideal building blocks for nanoscale electronics and optoelectronics. In particular, NCs composed of GaN and related group III-N alloys, having a huge potential for new devices, attract much attention recently. The aim of this paper is to report on our developments in MBE growth of GaN NCs on $\mathrm{Si}(111)$ substrates and on their detailed structural characterization. Preliminary results on use of GaN NCs in gas sensor devices are presented.

\section{Experiment}

Riber Compact 21 MBE system equipped with elemental source of Ga and an Addon RF plasma source of active nitrogen was used for the growth. The growth started by deoxidation of Si (111) substrate followed by its exposure to a nitrogen flux at $790{ }^{\circ} \mathrm{C}$. As the result, the reflection high-energy electron diffraction (RHEED) pattern changed from initial $(7 \times 7)$ into uncompleted $(8 \times 8)$ being a fingerprint of epitaxial $\beta-\mathrm{Si}_{3} \mathrm{~N}_{4}$ phase formed on silicon [1] (see Fig. 1). Then, the substrate was cooled to $740^{\circ} \mathrm{C}$ and the Ga cell was opened to start growth of nanocolumns under highly nitrogen-rich conditions. No catalyst was used to induce nucleation of NCs. Figure 1c shows RHEED pattern observed during growth of NCs. Transition from 2D (Fig. 1a,b) into 3D (Fig. 1c) surface is clearly visible.

\section{Results and discussion}

Figure 2 shows scanning electron microscopy image of the sample after $90 \mathrm{~min}$ of growth. As seen, a dense ensemble of $\mathrm{GaN} \mathrm{NCs}$ is obtained. The $\mathrm{NCs}$ are $\approx 350 \mathrm{~nm}$ long with diameter of $20-30 \mathrm{~nm}$. They are

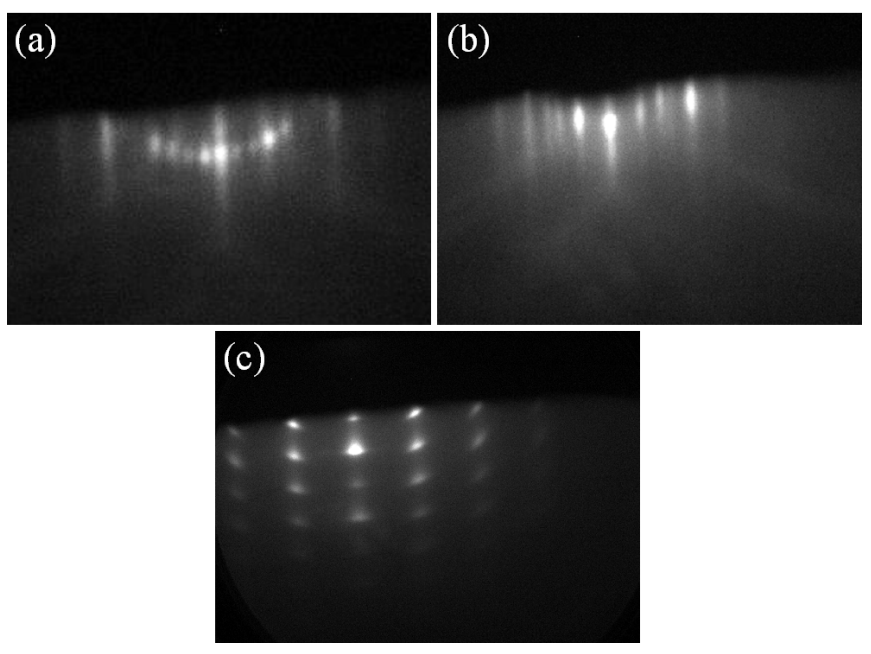

Fig. 1. RHEED pattern of a clean $\mathrm{Si}(111)$ substrate (a) after its nitridation (b) and during growth of GaN NCs (c).

homogeneously distributed and well oriented with the $c$-axis being perpendicular to the substrate. Density of nanocolumns is quite large $\left(\approx 300 \mu \mathrm{m}^{-2}\right)$. Therefore, even a small tilt of some of them leads to coalescence in their upper parts.

Figure 3 shows transmission electron microscopy (TEM) image of cross-section of GaN NCs on $\mathrm{Si}(111)$ substrate. Let us note absence of extended defects despite a large lattice mismatch between GaN and Si. Only occasionally some stacking faults can be found on TEM images. Moreover, $\approx 2 \mathrm{~nm}$ thick film of amorphous $\mathrm{Si}_{x} \mathrm{~N}_{y}$ between GaN and the substrate can be seen in Fig. 3 . Despite its presence GaN NCs are perfectly aligned to the $\mathrm{Si}(111)$. It is also noting worthy that neither a $\mathrm{GaN}$ wetting layer on the substrate nor $\mathrm{Ga}$ droplets that in principle could act as a catalyst on tips of GaN NCs are found. This indicates that NCs were grown spon- 


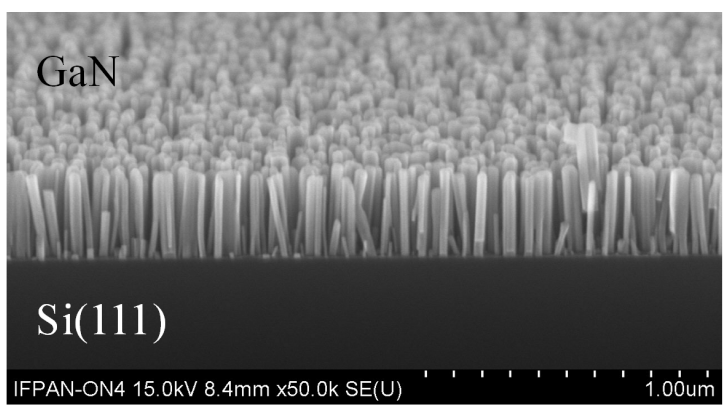

Fig. 2. SEM image of GaN NCs on $\mathrm{Si}(111)$ substrates.

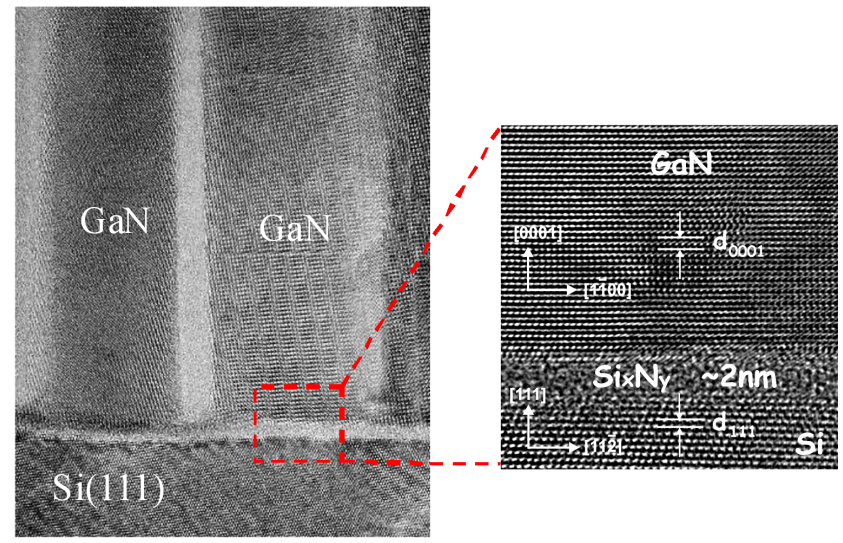

Fig. 3. TEM image of cross-section of $\mathrm{GaN} \mathrm{NCs}$ on Si(111) substrates.

taneously by the Volmer-Weber mechanism as discussed by Ristic et al. [2].

Finally, a test gas sensor device was prepared by alloying two ohmic contacts to a sample of GaN NCs on $\mathrm{Si}$ (Fig. 4a). Then, the sensor was put inside a test chamber and the resistance of the sample was measured while

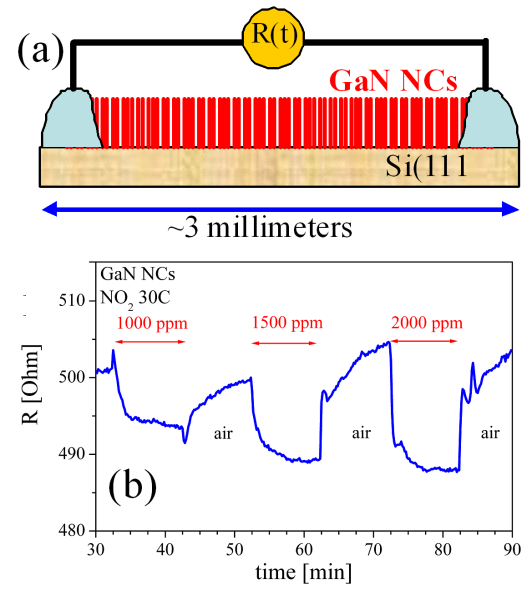

Fig. 4. Schematical drawing of GaN NCs gas sensor (a) and its response to presence of $\mathrm{NO}_{2}$ gas (b). the atmosphere in the chamber was continuously varied by precise dosing of a dedicated gaseous agent (see [3] for more experimental details). As an example, Fig. 4b shows that resistance of the sample is strongly influenced by a presence of $\mathrm{NO}_{2}$ gas and response of the sensor depends on the concentration of the gas injected to the chamber. Similarly, the GaN NCs device was found to be sensitive to presence of $\mathrm{NH}_{3}$ and hydrogen, but also to common solvents as ethanol or acetone. This shows a large potential of GaN nanocolumn structures for high sensitivity sensors. The work is in progress to increase sensitivity of the device by its miniaturization and optimization of its geometry.

\section{Summary}

Technology of plasma assisted MBE (PAMBE) growth of GaN NCs on $\mathrm{Si}(111)$ substrates has been developed. The nanocolumns nucleate spontaneously and grow vertically in the wurtzite crystalline form. Detailed structural characterizations indicate that they are nearly defect-free. Preliminary tests show that GaN NCs are promising building blocks for highly sensitive sensor devices.

\section{Acknowledgments}

This work was partly supported by the European Union within European Regional Development Fund, through grant Innovative Economy (POIG.01.01.02-00$-008 / 08$ NanoBiom).

\section{References}

[1] A. Le Louarn, S. Vezian, F. Semond, J. Massies, J. Cryst. Growth 311, 3278 (2009).

[2] J. Ristic, E. Calleja, S. Fernandez-Garrido, L. Cerutti, A. Trampert, U. Jahn, K.H. Ploog, J. Cryst. Growth 310, 4035 (2008).

[3] T. Pustelny, E. Maciak, Z. Opilski, M. Bednorz, Opt. Appl. 37, 187 (2007) 